\title{
Testing for haemoglobinopathies in Johannesburg, South Africa: A 30-year review
}

\author{
A Krause, ${ }^{1,2}$ MB BCh, PhD; T Wainstein, ${ }^{1}$ MSc (Med); F B Essop,,${ }^{1,2}$ MSc (Med); Q Goodyear, ${ }^{2}$ MMedSci \\ ${ }^{1}$ Division of Human Genetics, School of Pathology, Faculty of Health Sciences, University of the Witwatersrand, Johannesburg, South Africa \\ ${ }^{2}$ Division of Human Genetics, National Health Laboratory Service, Johannesburg, South Africa
}

Corresponding author: A Krause (amanda.krause@nhls.ac.za)

\begin{abstract}
Background. Haemoglobinopathies are seen mostly in regions where malaria occurs or has occurred, but population migration has resulted in affected individuals being identified in many countries globally. The first molecular genetics services for diagnostic testing and prenatal diagnosis were established, both worldwide and in South Africa (SA), for haemoglobinopathies.

Objective. To analyse the diagnostic service offered by the Division of Human Genetics, National Health Laboratory Service and University of the Witwatersrand, from 1983 to 2012.

Methods. A retrospective file analysis ( $N=1249)$ was performed for all individuals who had molecular genetic testing for $a$-thalassaemia, $\beta$-thalassaemia and sickle cell anaemia to examine indications for testing, population origins of patients and molecular genetics findings. Results. The $\alpha$-thalassaemia testing was requested predominantly to explain microcytic hypochromic haematological indices. Five $\alpha$-globin deletions were identified, the most common being the $-a^{3.7}$, in individuals of different ethnicities. For $\beta$-thalassaemia and sickle cell anaemia, most testing was performed for prenatal diagnosis purposes. For sickle cell anaemia, most prenatal tests were requested by African families. The $\beta$-thalassaemia families were mostly of Indian or Mediterranean origin. The most common mutation identified in all Indian groups was IVS1 nt5 (G>C) (c.92+5G>C) and in individuals from the Mediterranean, IVS1 nt110 (G>A) (c.93-21G>A).

Conclusion. The molecular genetics service for haemoglobinopathies in SA is comprehensive and specific to the needs of local ethnic groups. Clinically significant haemoglobinopathies occur at significant frequencies in specific high-risk ethnic groups. Appropriate screening programmes should be initiated so that genetic counselling and reproductive options can be offered.
\end{abstract}

S Afr Med 2013;103(12 Suppl 1):989-993. DOI:10.7196/SAMJ.7255

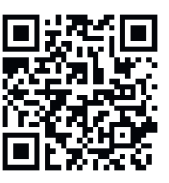

Haemoglobinopathies are considered the most common monogenic disorders. Clinically significant conditions are caused primarily by mutations in either the $\beta$ - or $\alpha$-globin genes. Mutations that cause quantitative deficiencies in $\beta$-globin result in $\beta$-thalassaemia, while qualitative variants result in $\beta$-globin variants, such as sickle cell anaemia. These are all inherited in an autosomal recessive manner. There are four $\alpha$-globin genes in the genome; thus mutations can occur in one, two, three or four genes, resulting in increasing severity of $\alpha$-thalassaemia, and a more complex inheritance pattern. ${ }^{[1,2]}$

Haemoglobinopathies are common mainly as a result of heterozygotes having a selective advantage against malaria. Their geographical distribution is thus generally in tropical and subtropical areas of the world, where malaria is/was previously endemic. With population migration, however, haemoglobinopathies are encountered in significant frequencies in many countries globally, particularly those with large migrations from Africa, the Middle East, the Indian subcontinent, South-East Asia, and the Mediterranean. Haemoglobinopathies thus represent a significant and increasing health burden in many parts of the world. ${ }^{[3]}$

While seasonal transmission of malaria has occurred in South Africa (SA), particularly in the north, the country is mostly situated out of the malaria belt. However, there have been a number of earlier and more recent migrations of groups of people into the area, with their respective haemoglobinopathies. The south-eastern Bantu migrated into southern Africa some 2000 years ago and have high frequencies of the $-a^{3.7}$ chromosome, suggesting it may be the oldest malaria protective trait in Africa. ${ }^{[4]}$ From the early 1860s until 1911, thousands of Indians were recruited to work on farms in Natal under the indentured labour system. They were accompanied by immigrants seeking opportunities as traders or craftsmen. ${ }^{[5]}$ More recently, families from the Mediterranean, north Africa, Asia and the African subcontinent have moved to SA. Thus, haemoglobinopathies are seen in diverse populations in SA, broadly reflecting their origins.

Further, the globin genes were among the first human genes cloned in the recombinant DNA era and they served as model genes for the establishment of genetic services worldwide, with the first molecular prenatal diagnosis being offered in 1978 for sickle cell anaemia. ${ }^{[6]}$ In SA, the first molecular genetics services for diagnostic testing and prenatal diagnosis were also set up for haemoglobinopathies, in the early 1980s, with the foresight and guidance of Professor Trefor Jenkins. The first prenatal diagnosis in SA was performed for an Indian couple at risk for sickle cell anaemia. ${ }^{[7]}$ Shortly thereafter, the first chorionic villus biopsy was performed in SA for an Indian couple at risk for $\beta$-thalassaemia. ${ }^{[8]}$ The laboratory in Johannesburg has continued to develop and expand its molecular diagnostic service for haemoglobinopathies, initially using Southern blotting and linked marker analysis, converting to polymerase chain reaction (PCR)-based mutation detection, with allele-specific oligonucleotides and amplification refractory mutation system (ARMS). Later a multiplex PCR (mPCR) system for $\alpha$-thalassaemias and gene sequencing and multiplex ligation probe amplification (MLPA) for $\beta$-globin gene cluster analysis was introduced.

\section{Objective}

To analyse the diagnostic service offered by the Division of Human Genetics (DHG), School of Pathology, University of the Witwatersrand, over the past 30 years, in terms of indications for testing, population 
profiles of patients and molecular genetics findings. The DHG was initially part of the South African Institute for Medical Research, but now falls within the National Health Laboratory Service (NHLS).

\section{Methods}

The data collected from molecular genetics files were analysed for all patients referred for haemoglobinopathy testing to the DHG ( $N=1249)$. These patients came from 465 families. Files are allocated a sequential family number, which incorporates a code defining whether they were referred for $\alpha$-thalassaemia, $\beta$-thalassaemia or sickle cell anaemia testing. There are some redundancies and anomalies in the system as families are usually given two codes if the original test request includes more than one haemoglobinopathy. In addition, they may occasionally have a diagnosis different from the original referral. Family members (including spouses) are all linked under the same alpha-numeric code. Files were available dating back to 1983 for $\alpha$-thalassaemia, 1984 for $\beta$-thalassaemia and 1987 for sickle cell anaemia. Only files with molecular genetic data were included in the analysis. If no such data were available, or if samples were not analysed, files were excluded. All files until 31 December 2012 were included.

The characteristics of the sample, and the indications for testing were assessed, as well as the molecular genetic findings.

\section{Results \\ a-thalassaemia}

Altogether, 292 individuals were tested for $\alpha$-thalassaemia, including 256 unrelated individuals from 241 families. The majority of referrals to the molecular diagnostic laboratory were tested as single individuals, with the most common indication for testing being unexplained microcytic hypochromic indices on full blood count (FBC) analysis. Individuals were initially investigated for common $\alpha$-globin deletions by the Southern blotting technique. An mPCR assay ${ }^{[9]}$ was introduced in 2001, which detects six common deletions in one assay.

Five common $\alpha$-globin deletions were identified with 10 genotypes in 132/256 (51.6\%) unrelated individuals tested (Table 1). The most common deletion identified was the $-\mathrm{a}^{3.7}$ in individuals from many different ethnic groups. In a further 124 individuals (48.4\%), no mutation was found. Despite the request form asking for haematological results (FBC, haemoglobin $(\mathrm{Hb})$ electrophoresis, iron studies) so that haematological and genetic results could be correlated, some or all of this information was only available for 193/292 (66\%) individuals. In $129 / 193$ (67\%) individuals, the haematological results were broadly consistent with the genetic results, either because an $\alpha$-thalassaemia mutation was identified $(n=100)$ or a different genetic mutation was ultimately identified $(n=29)$. In the remaining $64 / 193$, genetic and haematology results were inconsistent. No $\alpha$-thalassaemia mutations were identified, but 53 individuals had microcytic hypochromic indices. However, for many, the haematological results were incomplete, so a full assessment was not possible.

There were three families in which a proband, with features suggestive of haemoglobin $\mathrm{H}(\mathrm{HbH})$ disease, was tested. Deletions of three a genes were identified in two of these families. In the third family, a $-(\alpha)^{20.5}$ deletion was identified, but not a second mutation (Table 1). In one of these families, the proband was an adult female of Mediterranean origin concerned about her children's risks of a significant haemoglobinopathy.

Only four prenatal tests were performed for $\alpha$-thalassaemia, two for a Chinese family, where both parents were carriers of a -- ${ }^{\text {SEA }}$ mutation and they had three previous stillborn infants with hydrops fetalis. One fetus was found to be a carrier, the other unaffected. The other two prenatal tests were inappropriate requests. In the first, fetal testing for $\alpha$-thalassaemia was requested on an amniotic fluid sample. The

\begin{tabular}{|c|c|c|}
\hline Genotype & $\begin{array}{l}\text { Unrelated } \\
\text { individuals } \\
(N=256), n\end{array}$ & Population groups \\
\hline$-\alpha^{20.5} / ?$ & 1 & Mediterranean* \\
\hline$-a^{3.7} /--$ MED & 1 & Mediterranean* \\
\hline$-\alpha^{3.7} /--{ }^{\text {SEA }}$ & 1 & $\operatorname{Asian}^{\dagger}$ \\
\hline$-a^{3.7} /-a^{3.7}$ & 52 & Black, Indian, ${ }^{\ddagger}$ mixed ancestry \\
\hline$-a^{3.7} /-a^{4.2}$ & 3 & Arab, Indian ${ }^{\ddagger}$ \\
\hline$-a^{4.2} /-a^{4.2}$ & 3 & Indian, ${ }^{\ddagger}$ mixed ancestry, unknown \\
\hline$-3.7 / \alpha \alpha$ & 57 & $\begin{array}{l}\text { Arab, black, Indian, }{ }^{\ddagger} \text { Mediterranean, }{ }^{*} \\
\text { mixed ancestry, white }\end{array}$ \\
\hline${ }_{--} \mathrm{SEA} / \alpha \alpha$ & 6 & $\operatorname{Asian}^{\dagger}$ \\
\hline$--^{4.2} / \alpha \alpha$ & 5 & Indian, ${ }^{\ddagger}$ unknown \\
\hline$-{ }^{\mathrm{MED}} / \alpha \alpha$ & 3 & Mediterranean, ${ }^{*}$ white, ${ }^{\dagger}$ unknown \\
\hline$\alpha \alpha / \alpha \alpha$ & $124^{\prime}$ & $\begin{array}{l}\text { Arab, Asian, }{ }^{\dagger} \text { black, Indian } \\
\text { Mediterranean, }{ }^{*} \text { mixed ancestry, white }\end{array}$ \\
\hline \multicolumn{3}{|c|}{ 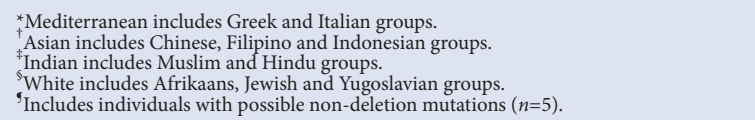 } \\
\hline
\end{tabular}

father was confirmed to be an $\alpha$-thalassaemia carrier, but the mother was shown to be a $\beta$-thalassaemia carrier after the amniocentesis had been performed. The second fetus, referred because of the presence of hydrops fetalis, was subsequently shown to have trisomy 21 .

In a consanguineous Indian Muslim family, two children had $\mathrm{HbH}$ disease. No deletions could be demonstrated. The parents shared a haplotype across the entire a-globin cluster and it was proposed that the children who were homozygous for the haplotype were homozygous for an $\alpha 2$ point mutation. ${ }^{[10]}$

There were four other families with possible non-deletion $a$ variants not identified with current testing. These were not pursued at the time because of cost and technical constraints, and the small number of patients for whom such testing would be indicated.

\section{Sickle cell anaemia}

Samples for a total of 319 individuals from 126 families were analysed for the sickle cell $\left(\beta^{s}\right)$ mutation, only one with known consanguinity. Of the 126 families, 116 were of African origin, four Indian, one mixed ancestry and five unknown. The 116 African families originated from at least 14 African countries in sub-Saharan Africa, and included 30 from the Democratic Republic of Congo, 16 from Angola, 10 from Nigeria and five from Zimbabwe. None originated from SA. It could not be determined whether the African patients were resident in SA or had only come to the country for their diagnostic procedures.

Altogether, 175 carrier tests and 31 clinical diagnostic tests were performed, many on parents of affected individuals as part of a workup for prenatal diagnosis. In 24 families, carrier screening was performed premaritally or at the time of other pregnancy testing (e.g. amniocentesis for advanced maternal age). In four families, confirmation of the clinical diagnosis was requested, where some of the clinical features were not entirely consistent with homozygosity for sickle cell anaemia. A single preclinical diagnosis was performed on a 6-month-old sibling of an affected individual.

There were 110 prenatal tests performed for 75 families, with 55 families having one prenatal test, 12 having two, four having three, two 


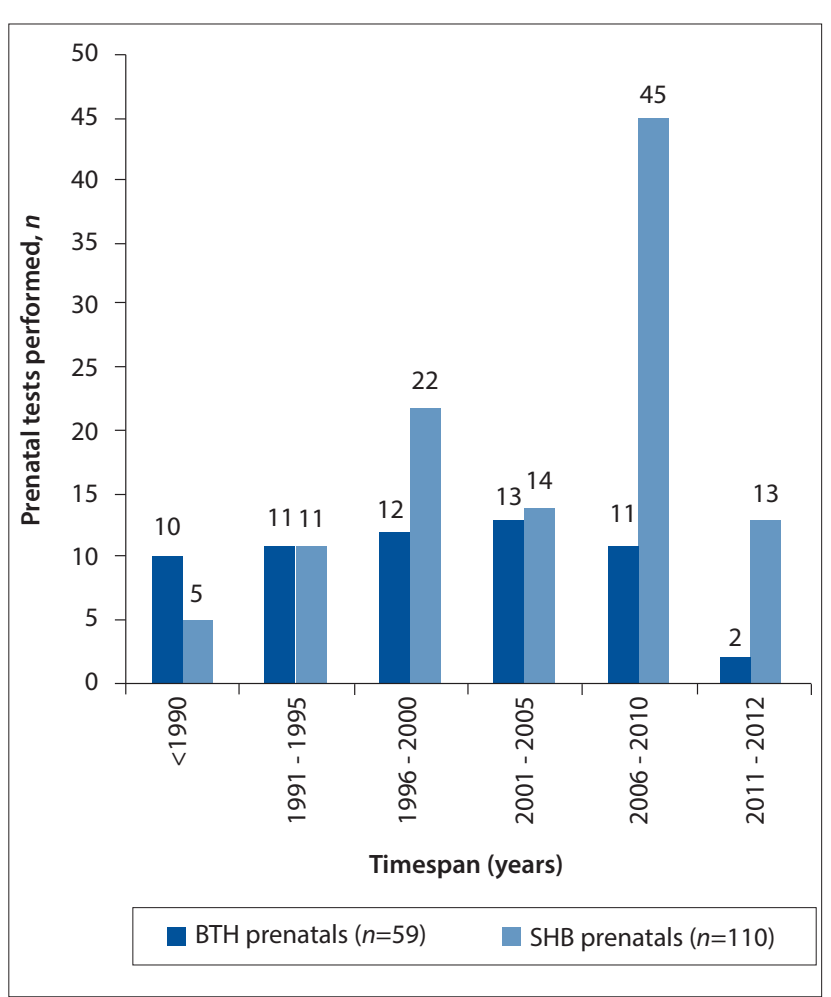

Fig. 1. $\beta$-thalassaemia (BTH) and sickle cell anaemia (sickle haemoglobinopathy (SHB)) prenatal tests performed over 5-year periods from 1988 to 2012.

families having four and one each having five and six tests, respectively. The 110 prenatal tests were performed on 58 amniotic fluid samples, 49 chorionic villus samples and three cordocenteses. The numbers, although fluctuating, have generally increased over the years, so that sickle cell anaemia is currently the most common indication for prenatal diagnosis by DNA testing in the laboratory (Fig. 1). The numbers of unaffected ( $n=29)$, carrier $(n=59)$, affected $(n=22)$ and no result $(n=1)$ fetuses did not differ from what would be expected based on Mendelian ratios. One erroneous report is known, where an affected fetus was misdiagnosed as a heterozygote, due to maternal tissue contamination of the specimen. Since then, from the year 2000, all heterozygous specimens have been checked routinely for maternal contamination. In 16 families a prenatal workup was requested but no prenatal test had yet been performed (including one family with non-paternity).

In addition, six fetuses were shown to have trisomies on routine karyotyping performed at the time of prenatal diagnosis; five had trisomy 21 and one trisomy 18, three being in mothers of advanced maternal age.

Three unusual probands were tested. In one, the proband was a heterozygote for the $\beta^{s}$ mutation. Clinically, she had features suggestive of homozygosity for sickle cell anaemia, with many sickling crises. No cis- or trans-variation in her $\beta$-globin genes could be identified. She was subsequently shown to have pyruvate kinase deficiency (Dr N Alli, personal communication). A second heterozygous proband also had clinical features of pseudoxanthoma elasticum and a third appeared to have a co-inherited non-deletion $\alpha$-globin variant.

\section{$\boldsymbol{\beta}$-thalassaemia}

A total of 416 individuals from 134 families were tested for $\beta$-thalassaemia. The majority of people (324 individuals from 84 families) were tested as part of a workup for prenatal diagnosis, where there was a previously affected child (70 families) or both parents had been identified as carriers haematologically (14 families). At least eight of these families were known to be consanguineous. For most of the families, however, information on consanguinity was not provided. The majority of families were from the two known high-risk population groups for $\beta$-thalassaemia in SA, namely individuals from the Indian subcontinent (62 families, of whom 39 were Muslim and 23 were Hindu) and individuals from Mediterranean countries (18 families). Three families were of mixed ancestry and one of unknown ethnicity. In the majority of families, both parents were $\beta$-thalassaemia carriers, so they were at risk of having children with thalassaemia major. There were also $13 \beta^{s} / \beta$-thalassaemia families included in the analysis (seven Indian, two Mediterranean, three African and one of mixed ancestry).

There were 59 prenatal tests performed for 43 families (including two $\beta^{\mathrm{s}} / \beta$-thalassaemia families), with 29 families having one test, 12 having two and two having three. Mendelian ratios of fetuses were as expected (14 unaffected, 27 heterozygotes, 12 affected). In six tests performed early on, full testing was not available, as either only one parental mutation was identified and/or linked markers were uninformative for one parent. Four fetuses were therefore considered at increased risk of a haemoglobinopathy, while two were at reduced risk. No errors were reported.

No trisomies were documented from the data available. Chromosome results were not available on all fetuses. The numbers of prenatal test requests have remained relatively constant over the last 25 years with $2-3$ requests per year (Fig. 1). Recently, two families have opted for pre-implantation genetic diagnosis. One has a healthy baby as a result. The other couple is currently going through the process. They would like to select an embryo for implantation, which is both unaffected for $\beta$-thalassaemia and a human leukocyte antigen (HLA) match for their affected child, so that the infant could be a suitable marrow donor for their affected child.

In 50 individuals, mutation screening was requested to clarify haematological findings. In 14 of these, both $\alpha$ - and $\beta$-thalassaemia testing were requested. As with $\alpha$-thalassaemia testing requests, in many cases haematological findings were not provided for interpretation and correlation. In $15 / 50$ individuals a $\beta$-thalassaemia mutation was identified. Four of these individuals were of Indian ancestry, four of mixed ancestry, five of Mediterranean and two of unknown ancestry.

The mutation profiles differed, based on the ethnic origins of the patients. Both the old nomenclature and the new Human Genome Variation Society (HGVS) nomenclature ${ }^{[11]}$ are used in Tables 2 and 3, but only the old nomenclature is used in the text for simplicity. Mutations identified in the Indian families (broadly divided into the two major religious groups) are shown in Table 2. The most common mutation identified in both Indian groups was IVS1 nt5 (G>C). In the Muslim group, two other mutations (IVS1 nt1 $(\mathrm{G}>\mathrm{T})$ and 619 bp deletion) together accounted for an additional 36\% of mutations identified. These two mutations are predominantly found in individuals from the Memon subgroup. In the Hindus, the codon 41 - 42 (-TTCT) mutation accounted for an additional $12.5 \%$ of mutations. The mutations found in the families of Mediterranean ancestry are shown in Table 3. The most common mutation $(77 \%)$ is the IVS1 $\mathrm{nt} 110(\mathrm{G}>\mathrm{A})$.

Interestingly all three mixed-ancestry families had at least one suspected deletion mutation. A haemoglobin $\mathrm{E}(\mathrm{HbE})$ mutation was identified in one family, and a codon $30(\mathrm{G}>\mathrm{C})$ mutation in another. In the proband heterozygous for the codon $30(\mathrm{G}>\mathrm{C})$ mutation, a deletion was confirmed with MLPA analysis (P102-B2 HBB probe $\mathrm{mix})^{[12]}$ and extends from exon 3 of the $\delta$-globin gene to intron 2 of the $\beta$-globin gene. The flanking probes, exon 1 of the $\delta$-globin gene and exon 3 of the $\beta$-globin gene were not deleted. The other two patients have not been screened. 
Table 2. $\beta$-thalassaemia mutations identified in unrelated individuals of Indian ancestry

\begin{tabular}{|c|c|c|c|}
\hline \multicolumn{2}{|c|}{ Mutations (nomenclature) } & \multirow{2}{*}{$\begin{array}{l}\text { Muslim patients } \\
(N=78), n(\%)\end{array}$} & \multirow{2}{*}{$\begin{array}{l}\text { Hindu patients } \\
(N=48), n(\%)\end{array}$} \\
\hline Old & HGVS $^{[11]}$ & & \\
\hline IVS1 nt5 (G>C) & c. $92+5 \mathrm{G}>\mathrm{C}$ & $32(41)$ & $28(58.3)$ \\
\hline IVS1 nt1 $(\mathrm{G}>\mathrm{T})$ & c. $92+1 \mathrm{G}>\mathrm{T}$ & $18(23.1)$ & $0(0)$ \\
\hline 619 bp deletion & 619 bp deletion & $10(12.8)$ & $1(2.1)$ \\
\hline Codon 41 - 42 (-TTCT) & c.124_127delTTCT & $1(1.3)$ & $6(12.5)$ \\
\hline Codon 8 - $9(+G)$ & c.27_28insG & $3(3.8)$ & $0(0)$ \\
\hline Codon 5 (-CT) & c.17_18delCT & $2(2.6)$ & $0(0)$ \\
\hline Codon $15(\mathrm{G}>\mathrm{A})$ & c. $47 \mathrm{G}>\mathrm{A}$ & $2(2.6)$ & $3(6.3)$ \\
\hline $\mathrm{HbE}$ & c. $79 \mathrm{G}>\mathrm{A}$ & $1(1.3)$ & $3(6.3)$ \\
\hline Codon $44(-\mathrm{C})$ & c. 135 delC & $1(1.3)$ & $0(0)$ \\
\hline Codon $30(\mathrm{G}>\mathrm{A})$ & c. $92 \mathrm{G}>\mathrm{A}$ & $1(1.3)$ & $0(0)$ \\
\hline Codon $30(\mathrm{G}>\mathrm{C})$ & c. $92 \mathrm{G}>\mathrm{C}$ & $1(1.3)$ & $1(2.1)$ \\
\hline $\mathrm{CAP}+1(\mathrm{~A}>\mathrm{C})$ & c. $-50 \mathrm{~A}>\mathrm{C}$ & $1(1.3)$ & $0(0)$ \\
\hline IVS1 nt2 (T>A) & c. $92+2 \mathrm{~T}>\mathrm{A}$ & $0(0)$ & $1(2.1)$ \\
\hline$-28(A>G)$ & c. $-78 \mathrm{~A}>\mathrm{G}$ & $0(0)$ & $1(2.1)$ \\
\hline Poly A $(\mathrm{T}>\mathrm{C})$ & c. $^{*}+110 \mathrm{~T}>\mathrm{C}$ & $0(0)$ & $1(2.1)$ \\
\hline HbD Punjab & c. $364 \mathrm{G}>\mathrm{C}$ & $1(1.3)$ & $0(0)$ \\
\hline Large deletion* & N/A & $2(2.6)$ & $0(0)$ \\
\hline Unknown $^{\dagger}$ & N/A & $2(2.6)$ & $3(6.3)$ \\
\hline
\end{tabular}

Table 3. $\beta$-thalassaemia mutations identified in unrelated individuals of Mediterranean ancestry

\begin{tabular}{|c|c|c|}
\hline \multicolumn{2}{|c|}{ Mutations (nomenclature) } & \multirow[b]{2}{*}{ Mediterranean patients $(N=35), n(\%)$} \\
\hline Old & HGVS $^{[11]}$ & \\
\hline IVS1 nt110 (G>A) & c. $93-21 \mathrm{G}>\mathrm{A}$ & $27(77.1)$ \\
\hline Codon $39(\mathrm{C}>\mathrm{T})$ & c. $118 \mathrm{C}>\mathrm{T}$ & $1(2.9)$ \\
\hline IVS1 nt1 (G>A) & c. $92+1 \mathrm{G}>\mathrm{A}$ & $1(2.9)$ \\
\hline IVS1 nt6 (T>C) & c. $92+6 \mathrm{~T}>\mathrm{C}$ & $2(5.7)$ \\
\hline IVS2 nt1 (G>A) & c. $315+1 G>A$ & $1(2.9)$ \\
\hline IVS2 nt745 (C>G) & c. $316-106 \mathrm{C}>\mathrm{G}$ & $1(2.9)$ \\
\hline Large deletion* & $\mathrm{N} / \mathrm{A}$ & $1(2.9)$ \\
\hline Unknown $^{\dagger}$ & N/A & $1(2.9)$ \\
\hline \multicolumn{3}{|c|}{$\begin{array}{l}\text { HGVS = Human Genome Variation Society; MLPA }=\text { multiplex ligation probe amplification; } \\
\text { ARMS = amplification refractory mutation system; } / \mathrm{A}=\text { not applicable. } \\
{ }^{*} \text { MLPA analysis shows a deletion including exon } 3 \text { of the } \delta \text { gene (but excluding exon } 1 \text { of the } \delta \text { gene), the entire } \beta \text { gene, and } \\
\text { extending } 0.8 \text { kb downstream of the } \beta \text { gene. }\end{array}$} \\
\hline
\end{tabular}

\section{Discussion}

Many different globin gene mutations have been identified through the diagnostic service of the DHG. They broadly reflect the population origins of the individuals represented. The laboratory has established a reputation for offering a comprehensive genetic service and thus both routine and atypical cases are referred.

\section{a-thalassaemia}

Our results emphasise the high frequency of a-thalassaemia mutations in many populations in SA. The distribution of the $\alpha$-thalassaemia mutations reflects the population origins of the individuals, similar to the situation worldwide. ${ }^{[1]}$ The majority of mutations are single a-gene deletions in heterozygous $\left(-\alpha^{3.7} / \alpha \alpha\right.$ or $\left.-\alpha^{4.2} / \alpha \alpha\right)$, homozygous $\left(-\alpha^{3.7} /-\alpha^{3.7}\right.$ or $\left.-a^{4.2} /-a^{4.2}\right)$ or compound heterozygous $\left(-a^{3.7} /-a^{4.2}\right)$ forms. Only a small number of individuals with both $\alpha$-genes deleted in cis (--/ $\alpha \alpha)$ were identified. Thus, not unexpectedly, only a few patients with $\mathrm{HbH}$ disease or hydrops fetalis have been documented in SA. Nevertheless, one of the first prenatal diagnoses for a haemoglobinopathy was performed for a couple of Chinese ancestry, where both parents carried a $-{ }^{\text {SEA }}$ mutation. A sizeable Chinese population of $\sim 250000$ - 300000 exists in Johannesburg. ${ }^{[13]}$ With an expected birth rate for $\mathrm{Hb}$ Bart's hydrops fetalis of $0.5-5 / 1000$ births and for $\mathrm{HbH}$ disease of $4-20 / 1000$ in the Chinese population, ${ }^{[1]}$ it is uncertain why there are so few referrals.

The majority of $a$-thalassaemia referrals were single individuals for the purpose of a-thalassaemia mutation detection. This usually stemmed from an inability to identify an explanation for microcytic hypochromic indices on FBC analysis with normal iron levels. Although these haemoglobinopathies do not cause clinically significant diseases, they cause concern for medical practitioners. DNA testing has proven to be helpful in confirming the presence of $\alpha$-thalassaemia mutations definitively, so that individuals are given appropriate management advice.

It is difficult to comment on the detection rate of the $\alpha$-thalassaemia multiplex assay as the indications for testing are often not provided, or only partial information is available. As a significant number of individuals are identified without any detectable $\alpha$-thalassaemia deletions, the possibility exists that non-deletion or rare deletion variants are being missed. However, the likelihood that these individuals are non$\alpha$-thalassaemia patients is more probable. Only a few individuals were identified who are likely to have a-globin mutations, but whose mutations remain unidentified.

Further, despite the request form asking for an $\mathrm{FBC}$ and $\mathrm{Hb}$ electrophoresis result to be provided, this did not occur in $\sim 40 \%$ of cases. In many, only partial information is provided. Thus it is difficult to assess the correlation between haematological and genetic findings. In addition, the haematological workup in itself is also inadequate in a number of cases. In many laboratories a qualitative assay is being used to quantitate $\mathrm{HbA}_{2}$ levels. Accurate $\mathrm{HbA}_{2}$ levels are important to distinguish $\alpha$ - from $\beta$-thalassaemia. These difficulties are reflected in the thalassaemia testing request, where in a notable number of cases, both $\alpha$ - and $\beta$-thalassaemia testing are requested, despite the fact that the majority should be distinguished haematologically. In many cases a non-genetic explanation for the haematological findings has not been completely excluded. 


\section{Sickle cell anaemia}

Sickle cell anaemia is the most common severe haemoglobinopathy in Africa, with high carrier rates in the tropical regions. ${ }^{[14]} \mathrm{It}$ is thus not surprising that the majority of patients referred to the DHG have origins in central Africa. Our records did not allow us to analyse how many patients only came to SA for prenatal testing, but are resident elsewhere in Africa and how many are resident locally, but, anecdotally, both scenarios do occur. The $\beta^{\mathrm{s}}$ mutation does occur at a low frequency in the northern parts of SA, ${ }^{[15]}$ but none of the families with an affected proband in this study were from SA.

The $\beta^{\mathrm{s}}$ mutation also occurs in India, and the carrier frequency is similar to that for $\beta$-thalassaemia in the SA Indian population. ${ }^{[10]}$ However, far fewer patients or families are seen with sickle cell anaemia, possibly because the patients are clinically mild and do not present with severe symptoms. Compound heterozygotes for $\beta^{\mathrm{s}}$ and $\beta$-thalassaemia mutations are diagnosed, and these are included in the $\beta$-thalassaemia analysis.

The majority of sickle cell anaemia tests are performed for families as part of a prenatal diagnostic workup. Sickle cell anaemia is the most common indication for prenatal diagnosis in the DHG, and the number of prenatal tests performed annually appears to be increasing.

Although some carrier testing for the $\beta^{s}$ mutation was performed by DNA analysis, this should not be performed routinely and is generally inappropriate. Qualitative $\mathrm{Hb}$ analysis remains the method of choice and is cheaper. In rare cases, molecular analysis may assist in resolving a complex case.

\section{$\beta$-thalassaemia}

As would be expected, $\beta$-thalassaemia testing was mostly performed for prenatal diagnosis purposes for families who were known to be at risk of a severe haemoglobinopathy. Early on, linked marker analysis was used, but this has gradually been replaced almost entirely by mutation analysis. MLPA has recently been introduced to identify large deletions in the $\beta$-globin gene cluster. Early on in the provision of the service, couples were sometimes only semi-informative with linked markers, thus only allowing for modification of fetal risk, based on information from the informative parent. Few unidentified mutations remain in verified $\beta$-thalassaemia families; thus almost all prenatal tests are offered with direct mutation analysis and high certainty. Carrier tests requested based on abnormal haematological findings and premarital screening of couples were less frequent indications for $\beta$-globin gene analysis.

The number of prenatal tests performed for $\beta$-thalassaemia has remained constant at 2 - 3 per year. Most couples were at risk for a child with $\beta$-thalassaemia, but some were at risk for $\beta^{s} / \beta$-thalassaemia.

The population specificity of $\beta$-thalassaemia mutations has been well documented in many populations. In most populations, a small number of mutations account for the majority of gene abnormalities in each group. ${ }^{[16]}$ In the Muslim group, three mutations account for $77 \%$ of mutations, and in the Hindu group, two mutations account for $71 \%$. In the Mediterranean group, one mutation accounts for $71 \%$.

Not only do populations' mutation spectra differ based on their geographical origins, but in addition, due to migrations of small groups of individuals and local founder effects, the mutation spectra may differ somewhat in people of similar origin. This is demonstrated in both of the SA high-risk populations - the Indian and Mediterranean groups. In the Indians, the most common mutation is the same in both the Muslim and Hindu groups. However, the 619 bp deletion and the IVS1 nt1 ( $\mathrm{G}>\mathrm{T})$ mutation, which together account for 36\% of mutations in the Muslims, are occasionally seen in Hindu people. Further, they appear at particularly high frequency in the Muslim Memon subgroup. Similarly the codon 41 - 42 (-TTCT) mutation, which accounts for $12 \%$ of mutations in the Hindu population, is only seen rarely in Muslims. In the early testing strategy, population identification was critically important as targeted mutation analysis was performed. However, as sequencing has become easier and less costly, this approach is no longer cost-effective and in most cases mutation identification is now achieved through sequencing. Targeted tests are, however, useful for prenatal testing.

\section{Conclusion}

The molecular genetics service offered to individuals in SA with haemoglobinopathies has improved technically and a wide knowledge base has developed over time, so that a comprehensive service specific to the needs of the local population is provided. Genetic testing is helpful to confirm diagnoses, particularly when they are complex, and for carrier testing and prenatal diagnosis. Prenatal diagnosis remains the most common indication for testing.

Comprehensive haematological assessment of patients should be performed prior to genetic analysis. These results, together with the ethnic group of the patient and information on consanguinity, should be passed on to the laboratory to ensure optimal testing and interpretation of the genetic data. This would prevent overinvestigation and result in the best possible management of individuals with haemoglobinopathies.

Although clinically significant haemoglobinopathies are not the most common monogenic disorders in SA, they do occur at significant frequencies in high-risk minority ethnic groups. Their treatment is expensive and will impact on health budgets, particularly if immigration from high-risk geographical areas increases. Further, with integration into the local communities, it will be increasingly difficult to direct testing at high-risk groups. It is important that appropriate screening programmes are initiated, so that high-risk couples are identified and appropriate genetic counselling and reproductive options are offered.

Acknowledgments. The authors acknowledge the clinicians and counsellors who referred these patients to the DHG, and the staff members of the Molecular Genetics Laboratory who have performed many tests over the years.

\section{References}

1. Galanello R, Cao A. Alph $\alpha$-Thalassaemia. In: Pagon RA, Adam MP, Bird TD, et al, eds. GeneReviews. Seattle: University of Washington, 1993-2013. http://www.ncbi.nlm.nih.gov/books/NBK1435/ (accessed 11 October 2013).

2. Cao A, Galanello R, Origa R. Beta-thalassaemia In: Pagon RA, Adam MP, Bird TD, et al, eds. GeneReviews. Seattle: University of Washington, 1993-2013. http://www.ncbi.nlm.nih.gov/books NBK1426/ (accessed 11 October 2013).

Weatherall DJ. Hemoglobinopathies worldwide: Present and future. Curr Molec Med 2008;8(7):592599. [http://dx.doi.org/10.2174/156652408786241375]

Ramsay M, Jenkins T. $\alpha$-thalassaemia in Africa: The oldest malaria protective trait? Lancet 1984;324(8399):410. [http://dx.doi.org/10.1016/S0140-6736(84)90581-6]

Bhana S, Brain JB. Setting Down Roots: Indian Migrants in South Africa, 1860-1911. Johannesburg. Witwatersrand University Press, 1990.

Witwatersrand University Press, 1990 .
6an YW, Dozy AM. Antenatal diagnosis of sickle-cell anaemia by DNA analysis of amniotic-fluid cells. Lancet 1978;312(8096):910-912. [http://dx.doi.org/10.1016/S0140-6736(78)91629-X]
Let.

Ramsay M, Thomson JA, Jenkins T. Antenatal diagnosis of sickle-cell anaemia by means of DNA restriction analysis. S Afr Med J 1984;66(1):7-10.

8. Ramsay M, Levin SL, Rosendorff J, et al. Chorionic villus sampling for first trimester diagnosis of betathalassaemia. Report of the first South African case. S Afr Med J 1985;68(9):672-675.

Chong SS, Boehm CD, Higgs DR, et al. Single-tube multiplex-PCR screen for common deletional determinants of $\alpha$-thalassaemia. Blood 2000;95(1):360-362.

10. Krause A. Globin Genes of the South African Indian Population. PhD thesis. Johannesburg: University of the Witwatersrand, 1994.

1. den Dunnen JT, Antonarakis SE. Mutation nomenclature extensions and suggestions to describe complex mutations: A discussion. Human Mutat 2000;15(1):7-12 [http://dx.doi.org/10.1002/ (SICI) 1098-1004(200001) 15:1<7::AID-HUMU4>3.0.CO;2-N]

12. MRC-Holland. MLPA. http://www.mrc-holland.com/WebForms/WebFormMain.aspx (10 July 2013).

13. Park YJ. Recent Chinese migrations to South Africa: New intersections of race, class and ethnicity. In Park YJ. Recent Chinese migrations to South Africa: New intersections of race, class and ethnicity. In:
Rahimy T, ed. Representation Expression and Identity: Interdisciplinary Perspectives. Oxford: InterRahimy T, ed. Representation Exp
Disciplinary Press, 2009:153-168.

Disciplinary Press, 2009:153-168.
14. Nagel RL, Fleming AF. Genetic epidemiology of the $\beta^{\text {s }}$ globin gene. Baillière's Clin Haematol 1992:5(2):331-365.

1992;5(2):331-365.
Jenkins T. Genetic Pol

15. Jenkins T. Genetic Polymorphisms of Man in Southern Africa. MD thesis. London: University of London, 1972.

6. Old JM. Hemoglobinopathies: Community Clues to Mutation Detection. In: Elles R, ed. Molecular Diagnosis of Genetic Diseases. New Jersey: Humana Press, 1996:169-183.

Accepted 22 August 2013. 